\title{
Mechanisms of motor symptom improvement by long-term Tai Chi training in Parkinson's disease patients
}

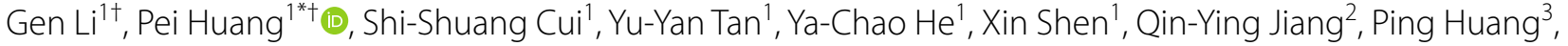
Gui-Ying He${ }^{1}$, Bin-Yin $\mathrm{Li}^{1}, Y u-X i n \mathrm{Li}^{2}$, Jin X $\mathrm{u}^{2}$, Zheng Wang ${ }^{4^{*}}$ and Sheng-Di Chen ${ }^{{ }^{*}}$

\begin{abstract}
Background: Tai Chi has been shown to improve motor symptoms in Parkinson's disease (PD), but its long-term effects and the related mechanisms remain to be elucidated. In this study, we investigated the effects of long-term Tai Chi training on motor symptoms in PD and the underlying mechanisms.

Methods: Ninety-five early-stage PD patients were enrolled and randomly divided into Tai Chi $(n=32)$, brisk walking $(n=31)$ and no-exercise $(n=32)$ groups. At baseline, 6 months and 12 months during one-year intervention, all participants underwent motor symptom evaluation by Berg balance scale (BBS), Unified PD rating-scale (UPDRS), Timed Up and Go test (TUG) and 3D gait analysis, functional magnetic resonance imaging (fMRI), plasma cytokine and metabolomics analysis, and blood Huntingtin interaction protein 2 (HIP2) mRNA level analysis. Longitudinal selfchanges were calculated using repeated measures ANOVA. GEE (generalized estimating equations) was used to assess factors associated with the longitudinal data of rating scales. Switch rates were used for fMRI analysis. False discovery rate correction was used for multiple correction.
\end{abstract}

Results: Participants in the Tai Chi group had better performance in BBS, UPDRS, TUG and step width. Besides, Tai Chi was advantageous over brisk walking in improving BBS and step width. The improved BBS was correlated with enhanced visual network function and downregulation of interleukin-1 $\beta$. The improvements in UPDRS were associated with enhanced default mode network function, decreased L-malic acid and 3-phosphoglyceric acid, and increased adenosine and HIP2 mRNA levels. In addition, arginine biosynthesis, urea cycle, tricarboxylic acid cycle and beta oxidation of very-long-chain fatty acids were also improved by Tai Chi training.

Conclusions: Long-term Tai Chi training improves motor function, especially gait and balance, in PD. The underlying mechanisms may include enhanced brain network function, reduced inflammation, improved amino acid

\footnotetext{
*Correspondence: hp12585@rjh.com.cn; zheng.wang@pku.edu.cn; chensd@rjh.com.cn

${ }^{\dagger}$ Gen Li and Pei Huang have contributed equally to this work

${ }^{1}$ Department of Neurology and Institute of Neurology, Ruijin Hospital

Affiliated to Shanghai Jiao Tong University School of Medicine,

Shanghai 200025, China

${ }^{4}$ School of Psychological and Cognitive Sciences, Beijing Key Laboratory

of Behavior and Mental Health, IDG/McGovern Institute for Brain

Research, Peking-Tsinghua Center for Life Sciences, Peking University,

Beijing 100871, China

Full list of author information is available at the end of the article
}

(c) The Author(s) 2022. Open Access This article is licensed under a Creative Commons Attribution 4.0 International License, which permits use, sharing, adaptation, distribution and reproduction in any medium or format, as long as you give appropriate credit to the original author(s) and the source, provide a link to the Creative Commons licence, and indicate if changes were made. The images or other third party material in this article are included in the article's Creative Commons licence, unless indicated otherwise in a credit line to the material. If material is not included in the article's Creative Commons licence and your intended use is not permitted by statutory regulation or exceeds the permitted use, you will need to obtain permission directly from the copyright holder. To view a copy of this licence, visit http://creativecommons.org/licenses/by/4.0/. The Creative Commons Public Domain Dedication waiver (http://creativeco mmons.org/publicdomain/zero/1.0/) applies to the data made available in this article, unless otherwise stated in a credit line to the data. 
metabolism, energy metabolism and neurotransmitter metabolism, and decreased vulnerability to dopaminergic degeneration.

Trial registration This study has been registered at Chinese Clinical Trial Registry (Registration number:

ChiCTR2000036036; Registration date: August 22, 2020).

Keywords: Parkinson's disease, Tai Chi, Motor symptoms, Mechanism, Brain network

\section{Background}

Parkinson's disease (PD) is the second most common neurodegenerative disease globally, characterized by bradykinesia, resting tremor and rigidity [1]. With progression of the disease, patients lose postural stability and have difficulty in gait and balance, causing frequent falls and disability in daily living [2]. Although some motor symptoms, such as tremor and rigidity, can be alleviated by drug therapy, some clinical features such as postural instability are less responsive to medication and need alternative treatments [3].

Physical exercise has been shown to improve mobility, gait, balance and quality of life in PD $[4,5]$. Tai Chi, brisk walking and tango dancing have demonstrated the highest level of evidence of efficacy, especially in improving postural stability [6-8]. Tai Chi, a mind-body exercise that utilizes continuous, curved, and spiral body movements with breathing control [9], can improve aerobic capacity, muscle strength, balance, and motor control, as well as reducing stress and anxiety in older adults [10]. Evidence from randomized controlled trials by Fuzhong Li et al. shows improvement of maximal excursion, direction control, gait velocity and quality of life after 6-month Tai Chi training in PD patients [6, 11]. However, previous studies focusing on Tai Chi training only showed shortterm (up to 6 months) benefits for PD patients. Owing to the progressive nature of $\mathrm{PD}$, the long-term effects of such interventions should be concerned.

More importantly, the beneficial mechanisms of Tai Chi remain unclear. Evidence based on animal studies of neurodegenerative diseases shows that physical exercise can improve the production of neurotrophic factors, neurotransmitters, and hormones, promoting processes such as synaptic plasticity, neurogenesis, angiogenesis, and autophagy [12]. Several studies focusing on the mechanisms of Tai Chi in older adults have shown improved brain metabolism and muscle energetics using brain ${ }^{1} \mathrm{H}$ magnetic resonance spectroscopy (MRS) and muscle ${ }^{31} \mathrm{P}$ MRS [13], as well as enhanced default mode network (DMN) connectivity using resting-state functional magnetic resonance imaging (fMRI) [14]. However, the mechanisms of Tai Chi training in PD patients have not been investigated.

fMRI and blood biomarker tests will probably give us a deep insight into the mechanisms of Tai Chi in PD.
Resting-state fMRI is widely used to explore brain function and neuroplasticity at the macro level. In addition, molecular biomarkers in the blood of PD patients, which can reflect pathogenesis and disease progression, also provide ways to study mechanisms of Tai Chi $[15$, 16]. Previous animal studies showed that exercise might benefit PD patients through inhibiting oxidative stress, repairing mitochondrial damage, and promoting the production of growth factors [4]. Huntingtin interaction protein 2 (HIP2) is an E2 ubiquitin-conjugating enzyme associated with neurodegenerative diseases. Decreased expression of HIP2 has been reported in the blood [1719] and the substantia nigra of PD patients [20]. Reduction of HIP2 expression could cause motor function impairment and increase vulnerability to dopaminergic degeneration in PD models [21].

In this study, we conducted a one-year randomized controlled trial to investigate the long-term effect of Tai Chi training on motor symptoms of PD and explore the underlying mechanisms by fMRI and blood biomarker (including cytokines, metabolomics and HIP2 mRNA) analysis.

\section{Methods}

\section{Participants}

Ninety-five early-stage (Hoehn-Yahr 1-2.5) PD patients (50-80 years old) were recruited. The medication was stable for at least 3 months before recruitment and not changed during follow-up unless necessary due to disease progression. This study was approved by Ruijin Hospital Ethic Committee of Shanghai Jiao Tong University School of Medicine on December 4, 2014. This study has been registered at Chinese Clinical Trial Registry (Registration number: ChiCTR2000036036; Registration date: August 22, 2020). PD was diagnosed by two movement disorder specialists (SDC, YYT). All of the PD patients met both the PD diagnostic criteria of United Kingdom Brain Bank [22] and Movement Disorders Society [23]. All participants provided written informed consents.

\section{Randomization}

After recruitment, participants were randomly assigned to Tai Chi $(n=32)$, brisk walking $(n=31)$ and control $(n=32)$ groups without stratification. The randomization method is to draw lots. Then, 12-month exercise 
intervention (Tai Chi, brisk walking or non-exercise) with strict quality control was introduced. Details could be seen in Additional file 1.

\section{Assessments}

Berg Balance Scale (BBS), Unified PD rating scale (UPDRS), Time Up and Go test (TUG) and spatial 3D gait analysis were used to assess motor symptoms, gait and balance of PD patients. Assessments were performed at baseline, 6 months and 12 months during the intervention on medication.

\section{Investigation of mechanisms}

fMRI, plasma cytokines and metabolomics, and the blood HIP2 mRNA level were assessed at baseline, 6 months and 12 months. Details are provided in Additional file 1.

\section{Statistical analysis}

Statistical analysis was performed using R (version 3.5.1), RStudio (version 1.1463) and related packages. fMRI data were analyzed using the MATLAB R2018a (version 9.4.0.813654, MathWorks, Inc.) software.

All analyses about clinical measurements were conducted on an intention-to-treat basis. Analysis of variance (ANOVA) was used to compare numerical demographic information. Pearson Chi-square test or Fisher test was used for analysis of categorical demographic information. Shapiro-Wilk normality test was used to assess the normality of variables. Independentsample $t$-tests (with 95\% confidence intervals) were used to compare group means. Data were presented in the way of between-group differences. The longitudinal selfchanges were analyzed with repeated measures ANOVA. AI-Therapy Statistics ${ }^{\text {BETA }}$ (https://www.ai-therapy.com/ psychology-statistics/power-calculator) was used to calculate the statistical power.

To analyze longitudinal fMRI data, GroupICAT (v4.0a, The MIND Research Network, Atlanta, GA) was used for group-level independent component analysis (ICA) to extract brain networks. Longitudinal changes of fMRI data were reflected by switching rate according to the protocol given by Pedersen et al. [24], in which 25 brain nodes were analyzed and sliding-window analysis was used to see brain network dynamic changes. Thus, functional network dynamic connectivity was analyzed to obtain the switching rate of brain networks. Statistical methods such as LASSO (least absolute shrinkage and selection operator) were used for screening and feature selection of high latitude variables (switching rates of various brain networks). To make the data normally distributed, Log transformation was introduced to selected switching rates. Finally, linear regression $\left(y=\sum x\right)$ was performed between the change of rating scales (regarded as $\mathrm{y}$ ) and the selected switching rates (regarded as $\mathrm{x}$ ). Bayesian belief network was also introduced to confirm the causality of the associations. Finally, false discovery rate (FDR) correction was adopted.

GEE (generalized estimating equations) was used to assess the association of longitudinal data of rating scales. Mixed effect regression using restricted maximum likelihood method was introduced to analyze cytokine levels, HIP2 levels and metabolites. Bonferroni correction was used for multiple corrections. Pathway analysis was performed using MetaboAnalyst 4.0 platform. Enrichment analysis was done using MetaboAnalyst 4.0 platform referenced with the small molecule pathway database (SMPDB). FDR correction was used for multiple correction. Detailed information is provided in Additional file 1.

\section{Results}

\section{Baseline characteristics of participants}

Ninety-five PD patients were recruited in this study, including 32 in the Tai Chi group, 31 in the brisk walking group, and 32 in the control group (see Fig. 1 for a flowchart of recruitment). All of them are of Han ethnicity, and none presented either on-off phenomenon or dyskinesias. The three groups were matched in age, sex, disease duration and education. Sixty-six patients finished 6-month and 12-month follow-ups. Detailed demographic information is shown in Table 1. There were no differences in the levodopa equivalent daily dosage (LEDD) at baseline and at 12-month visit among the groups.

\section{Clinical improvements}

The PD patients in the Tai Chi group showed significant improvements in BBS at 6 months and 12 months (6 months: $P=0.006,12$ months: $P=0.044, P$ values were based on between-group differences in longitudinal mean changes of score), compared to the control group. Compared to brisk walking, Tai Chi had more advantages in improving BBS scores (at 6 months: $P=0.005$, 12 months: $P=0.022$ ) (Additional File 2: Table S1; Fig. 2a).

Regarding UPDRS and TUG, the Tai Chi group showed significantly greater improvement in UPDRS at 12 months compared to controls (UPDRS total score: $P=0.015$, UPDRS-III: $P<0.001$ ), as well as significantly greater improvement in TUG at 6 and 12 months compared to control (6 months: $P=0.017,12$ months: $P=0.011)$. Tai Chi also significantly improved patients' step width compared to controls at 6 and 12 months (severer side, 6 months: $P=0.002,12$ months: $P<0.001$; milder side, 6 months: $P<0.001,12$ months: $P<0.001$ ). When compared to brisk walking, the Tai Chi group 


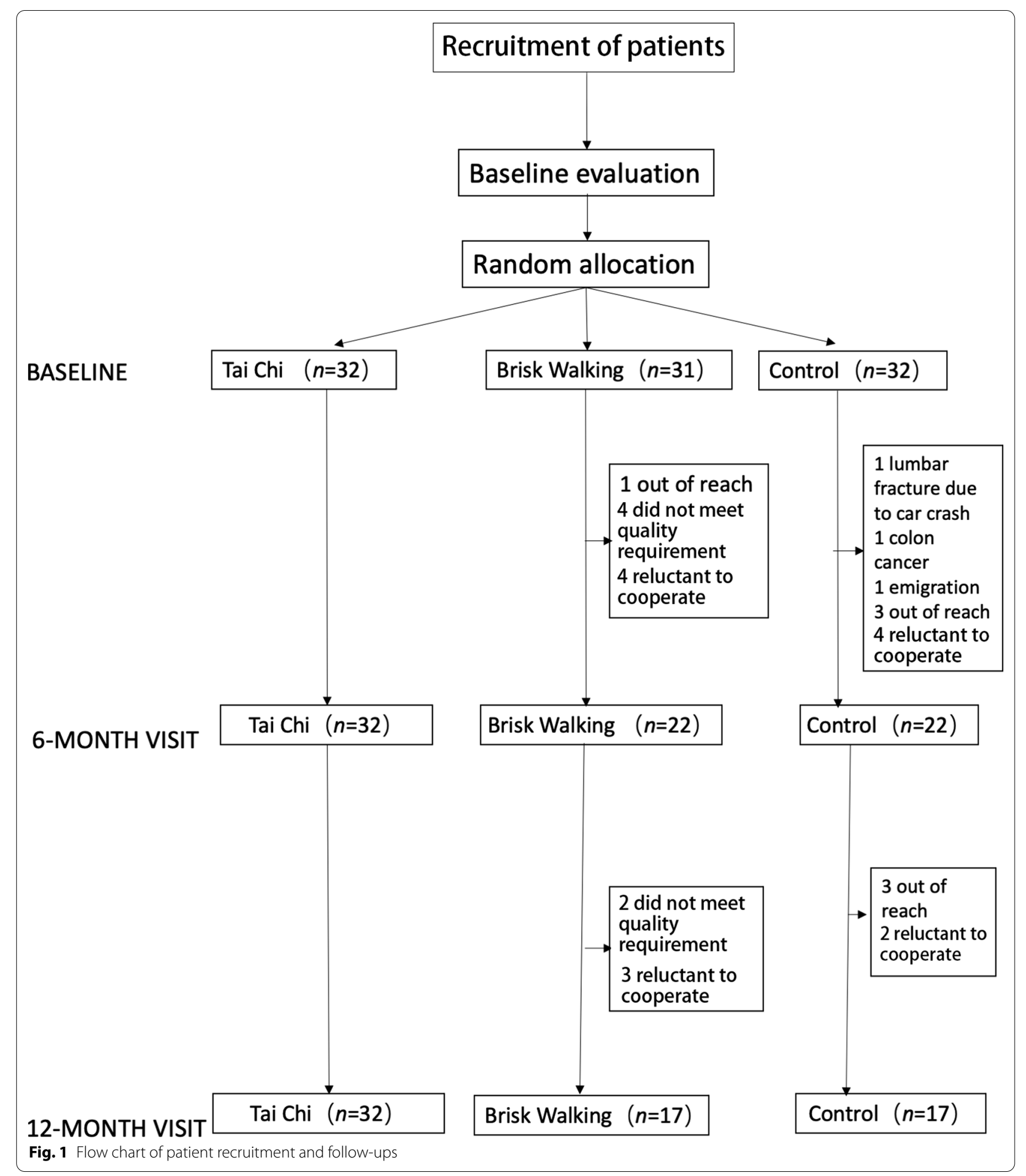

also showed greater improvement in step width (severer side, 6 months: $P=0.03,12$ months: $P=0.03$; milder side, 6 months: $P=0.004,12$ months: $P=0.111)$. Statistical powers were above 0.05 (BBS: 0.052; UPDRS total score:
0.059; UPDRS-III: 0.050; TUG: 0.061). (Additional file 2: Table S1, Fig. 2b-d). 
Table 1 Demographic information of participants

\begin{tabular}{|c|c|c|c|c|}
\hline & Tai Chi Group ( $n=32)$ & $\begin{array}{l}\text { Brisk Walking Group } \\
(n=31)\end{array}$ & Control Group $(n=32)$ & $P$ value \\
\hline Sex, female, $n(\%)$ & $15(46.88)$ & $9(29.41)$ & $13(41.18)$ & 0.500 \\
\hline Age at baseline (mean $\pm S D$ ) & $62.7(5.51)$ & $61.9(5.64)$ & $61.9(6.76)$ & 0.400 \\
\hline Education, years (mean $\pm S D$ ) & $13.60(2.71)$ & $13.10(2.57)$ & $12.40(2.83)$ & 0.472 \\
\hline History of hypertension, $n$ (\%) & $7(21.88)$ & $3(11.76)$ & $5(17.65)$ & 0.800 \\
\hline History of diabetes mellitus, $n(\%)$ & $1(3.13)$ & $0(0.00)$ & $1(3.13)$ & 1.000 \\
\hline History of smoking, $n(\%)$ & $2(6.25)$ & $2(6.25)$ & $2(6.25)$ & 0.600 \\
\hline Family history, $n(\%)$ & $8(25.00)$ & $5(17.65)$ & $3(11.76)$ & 0.600 \\
\hline Tremor dominant, $n(\%)$ & $22(68.75)$ & $22(64.71)$ & $17(58.82)$ & 0.800 \\
\hline Disease duration (mean $\pm S D$ ) & $5.91(4.01)$ & $3.82(1.87)$ & $4.32(2.46)$ & 0.082 \\
\hline Hoehn-Yahr staging, n (\%) & & & & 0.097 \\
\hline 1.0 & $9(28.13)$ & $8(25.81)$ & $1(3.13)$ & \\
\hline 1.5 & $5(15.63)$ & $7(22.58)$ & $11(34.38)$ & \\
\hline 2.0 & $13(40.63)$ & $13(41.94)$ & $13(40.63)$ & \\
\hline 2.5 & $5(15.63)$ & $3(9.68)$ & $7(21.88)$ & \\
\hline LEDD at baseline (mean \pm SD) & $326(197)$ & $260(174)$ & 347 (109) & 0.800 \\
\hline$\triangle \mathrm{LEDD}($ mean $\pm \mathrm{SD})$ & $56.33(91.68)$ & $39.71(83.30)$ & $57.21(107.24)$ & 0.939 \\
\hline
\end{tabular}

$\angle E D D$ levodopa equivalent daily dosage; $N$ number; $S D$ standard deviation

$\triangle L E D D$ LEDD at 12 months minus LEDD at baseline

\section{fMRI switch rates}

The better performance in BBS in the Tai Chi group compared with the control was associated with the change of visual network $(\mathrm{VN})(P=0.044)$. The improvements in UPDRS total score $(P=0.023)$ and UPDRS-III $(P=0.006)$ of PD patients were associated with enhancement of DMN connectivity after Tai Chi training (Additional File 2: Table S2; Fig. 2e-h).

\section{Cytokines}

The levels of interleukin (IL)- $1 \beta$ (6 months: $P=0.013$, 12 months: $P=0.028)$, IL-5 (6 months: $P=0.028$, 12 months: $P=0.248)$, IL-7 (6 months: $P=0.032$, 12 months: $P=0.016)$ and IL-9 $(6$ months: $P=0.037$, 12 months: $P=0.019$ ) were significantly downregulated in the Tai Chi group compared with the control group (Additional file 2: Table S3).

The levels of IL-13 (6 months: $P=0.002,12$ months: $P=0.005$ vs control), platelet-derived growth factor-BB (6 months: $P=0.022,12$ months: $P=0.011$ $v s$ control), macrophage inflammatory protein-1 $\beta$ (MIP-1 $\beta$ ) (6 months: $P=0.003,12$ months: $P=0.008$ vs control) and monocyte chemoattractant protein-1 (MCP-1) (6 months: $P=0.032,12$ months: $P=0.06$ vs control) were stable in the Tai Chi group, but significantly increased in controls. Relatively stable levels of IL-13 (6 months: $P=0.019,12$ months: $P=0.021$ vs brisk walking group) and MCP-1 (6 months: $P=0.0007,12$ months: $P=0.022 v s$ brisk walking group) were seen in the Tai Chi group compared with the brisk walking group (Additional file 2: Table S3). In addition, at 6 months, upregulation and downregulation of MIP- $1 \alpha$ were seen in the control group and brisk walking group, respectively, while the MIP-1 $\alpha$ level did not change in the Tai Chi group $(P=0.006$ control $v s$ Tai Chi, $P=0.029$ brisk walking group $v s$ Tai Chi). But MIP-1 $\alpha$ returned to the baseline level in the three groups at 12 months (Additional file 2: Table S3).

Granulocyte-macrophage colony stimulating factor (GM-CSF) was upregulated in the Tai Chi group while being not changed in the control group or the brisk

(See figure on next page.)

Fig. 2 Changes of motor symptoms between groups and related fMRI changes. $\mathbf{a}-\mathbf{d}$ Changes of rating scales in a Berg Balance Scale, $\mathbf{b}$ UPDRS total Score, $\mathbf{c}$ UPDRS Part III, and $\mathbf{d}$ Timed Up and Go Test in the 3 groups. Data are shown as mean \pm SEM. e- $\mathbf{h}$ Neural networks associated with the longitudinal changes of rating scales from 12-month to baseline after Tai Chi training. Bright yellow indicates more positive association between neural networks and changes of rating scales. e Visual network associated with Berg Balance scale; $\mathbf{f}$ default mode network associated with UPDRS total score; $\mathbf{g}$ Ventral salient network associated with UPDRS total score, but the association was not significant; $\mathbf{h}$ default mode network associated with UPDRS Part III 

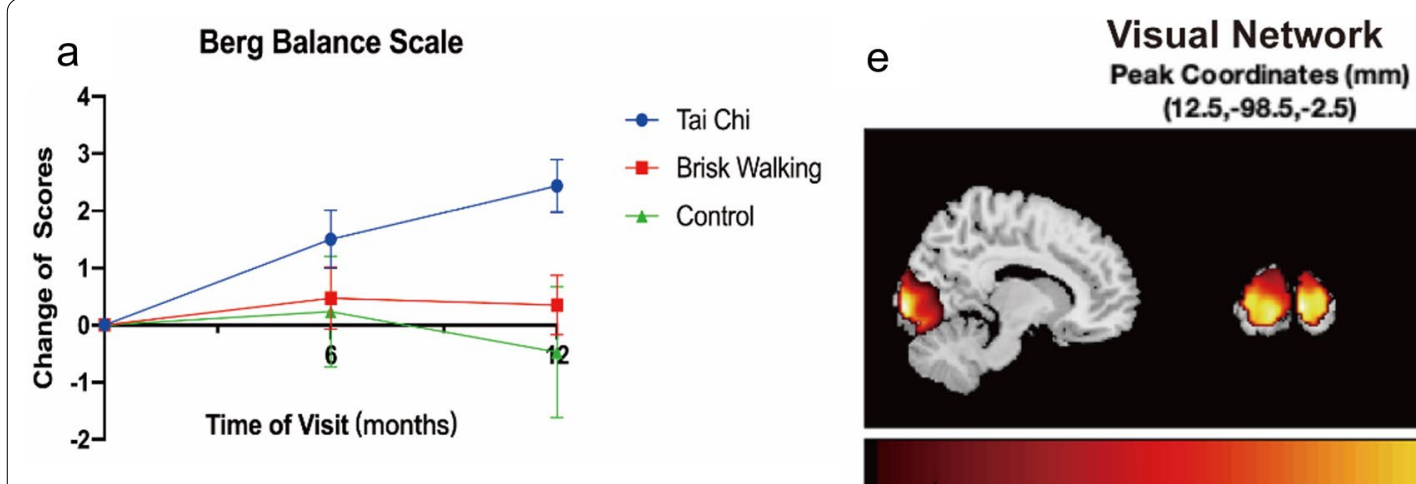

$(12.5,-98.5,-2.5)$
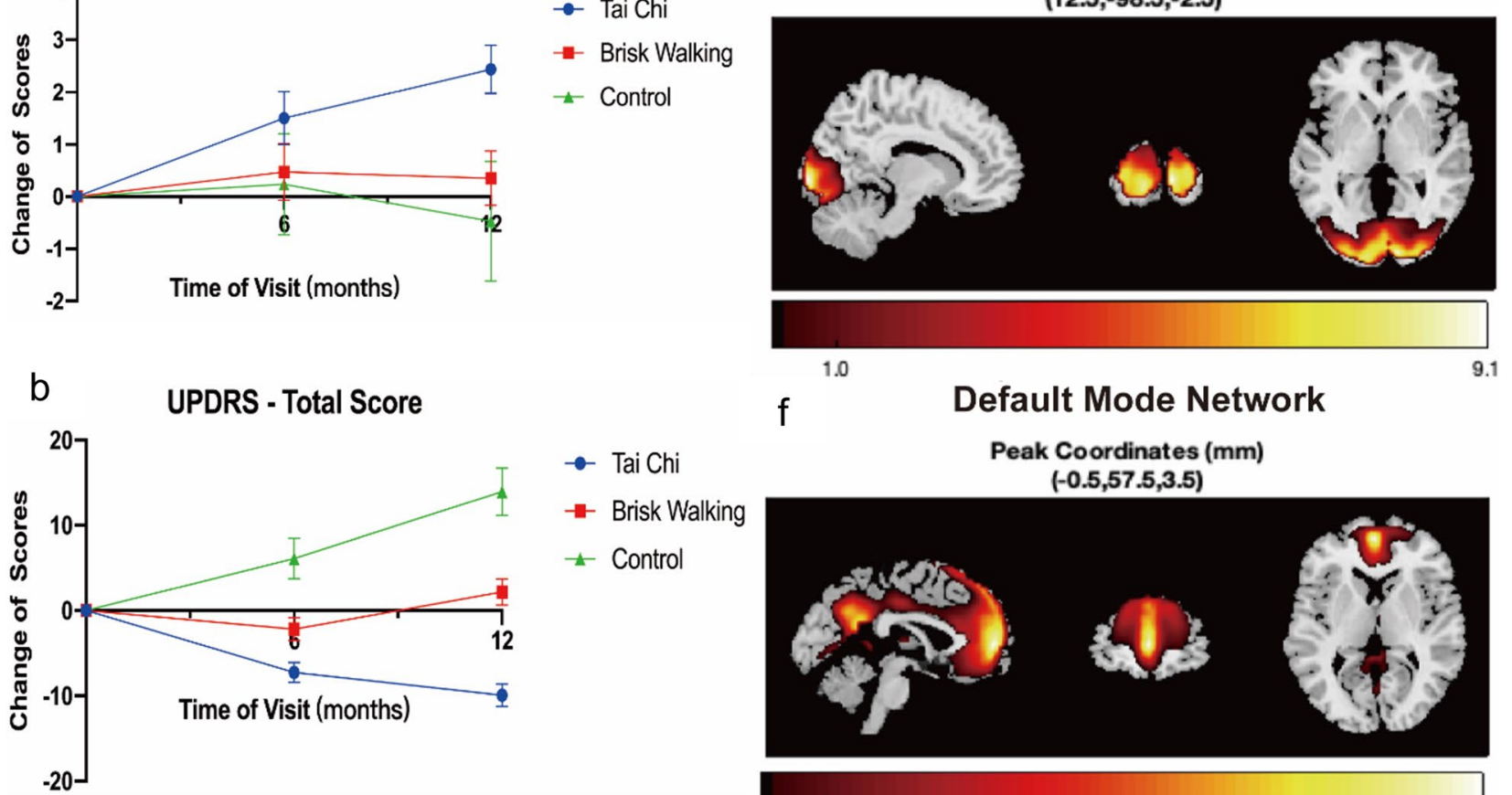

f
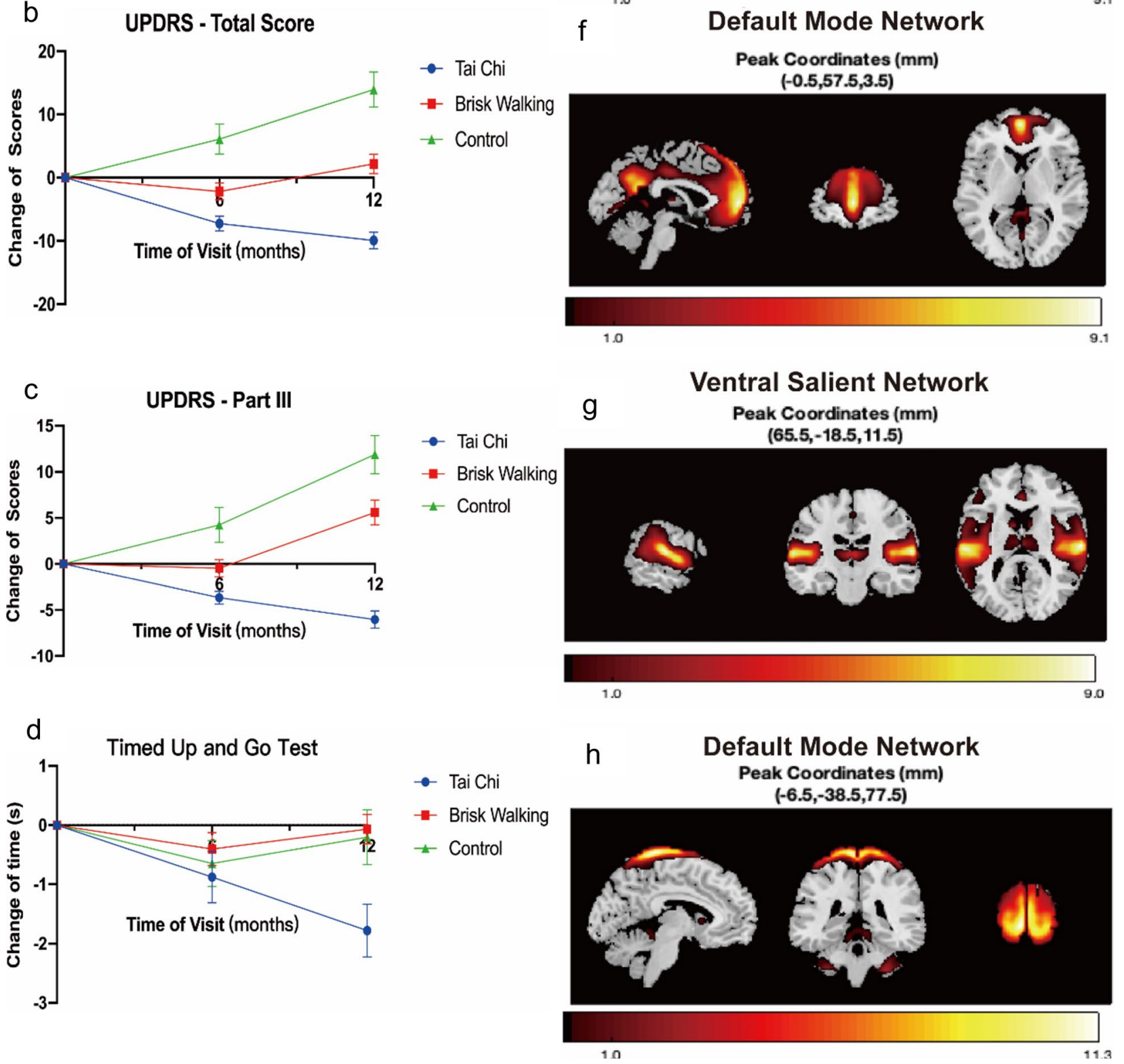

Fig. 2 (See legend on previous page.) 
walking group (Tai Chi vs control, 6 months: $P=0.005$, 12 months: $P=0.011$; Tai Chi $v s$ brisk walking, 6 months: $P=0.006,12$ months: $P=0.014$ ) (Additional file 2: Table S3).

Furthermore, by analyzing the association between changes of cytokines and changes of clinical presentation, we found that the downregulation of IL- $1 \beta$ was positively related to the improved BBS score $(P \leq 0.031)$ (Additional file 2: Table S4).

\section{Metabolomics}

We tested 123 metabolites, of which 27 metabolites showed a statistically significant change after Tai Chi training. After Bonferroni correction, 11 metabolites were left significant. Fumaric acid, $L$-aspartic acid and pyroglutamic acid were decreased after Tai Chi training only at 6 months $(P \leq 0.033)$. Downregulation of homocysteine and methionine sulfoxide, and upregulation of azelaic acid were seen in the Tai Chi group both at 6 months $(P \leq 0.005)$ and at 12 months $(P \leq 0.032)$. $L$-malic acid and 3-phosphoglyceric acid were downregulated, while $L$-fucose, adenosine and pipecolic acid were upregulated after Tai Chi training at 12 months $(P \leq 0.028)$ (Additional file 2: Table S5).

We also found several associations between metabolites and clinical presentations. The levels of $L$-malic acid, 3 -phosphoglyceric acid and adenosine were associated with the changes of UPDRS total score $(P \leq 0.043)$. The levels of $L$-malic acid, $L$-fucose, and pipecolic acid were associated with the changes of UPDRS-III $(P \leq 0.041)$ (Additional file 2: Table S6).

Pathway analysis showed group differences in arginine biosynthesis both at 6 months (Tai Chi vs control: $P=0.007$; Tai Chi $v s$ brisk walking: $P=0.006)$ and at 12 months (Tai Chi vs brisk walking: $P<0.001$ ) (Additional file 2: Table S7, Additional file 3: Fig. S1-S3). In enrichment analysis, significant group differences were found in urea cycle both at 6 months (Tai Chi vs control: $P=0.009$; Tai Chi vs brisk walking: $P=0.05)$ and at 12 months (Tai Chi vs brisk walking: $P<0.001$ ) (Additional file 2: Table S8, Additional file 3: Fig. S4-S6).

In association analysis, the tricarboxylic acid (TCA) cycle was correlated with BBS $(P=0.037)$, UPDRS total score $(P=0.002)$, and UPDRS-III $(P=0.014)$. Beta oxidation of very-long-chain fatty acids was relevant to UPDRS total score $(P=0.033)$ and UPDRS-III $(P=0.033)$ (Additional file 2: Table S9).

\section{HIP2 mRNA levels}

In the control group, expression of HIP2 mRNA showed a tendency of downregulation $(P=0.697)$. Compared to the control group, the HIP2 mRNA level was elevated after Tai Chi training for 6 months $(P<0.001)$ and
12 months $(P<0.001)$. Tai Chi training showed a tendency to be better in upregulating HIP2 mRNA level than brisk walking $(P=0.277)$. (Additional file 3: Fig. S7).

We found that the differences in UPDRS total score and UPDRS-III score were associated with the change of HIP2 mRNA level after Bonferroni correction $(P<0.005)$. The association between the difference in BBS score and the change of HIP2 mRNA level did not survive after Bonferroni correction (Additional file 2: Table S10).

\section{Discussion}

In this study, we found long-term beneficial effects of Tai Chi in improving balance and other motor symptoms in PD. Tai Chi improved BBS, UPDRS, TUG and step width, indicating its beneficial effects on motor symptoms (including gait and balance). Tai Chi performed better than brisk walking in improving BBS and step width.

More importantly, we explored the mechanisms underlying improvement of motor symptoms of PD after Tai Chi training. fMRI test revealed association between changes of BBS score and the switch of $\mathrm{VN}$, and a positive relationship between improvement of UPDRS and the function of DMN. Plasma cytokines IL-1 $\beta$, IL-5, IL-7, IL-9, IL-13, MCP-1, MIP-1a, and MIP-1 $\beta$ were relatively downregulated and GM-CSF was upregulated after Tai Chi training. Among them, the downregulation of IL- $1 \beta$ was positively related to the improved BBS scores. The decreased $L$-malic acid and 3-phosphoglyceric acid, and increased adenosine were associated with changes of UPDRS total score in PD after Tai Chi training, while downregulation of $L$-malic acid, and upregulation of $L$-fucose and pipecolic acid were related to changes of UPDRS-III. Arginine biosynthesis, urea cycle, TCA cycle and beta oxidation of very-long-chain fatty acids were also affected by Tai Chi. The HIP2 mRNA level was significantly elevated after Tai Chi training, and its change was correlated with the changes of UPDRS total score and UPDRS-III score in PD after Tai Chi training.

Our study found significant improvement of motor function (especially gait and balance) in PD patients after Tai Chi training, which was consistent with the results of previous studies [6, 11]. The mechanisms underlying the beneficial effects might be associated with the improved brain network function in PD patients. The $\mathrm{VN}$ is composed of bilateral striate and extrastriate visual areas [25]. Visual proprioceptive sensory conflict could influence gait and balance [26]. Visual cues can lessen the vestibular noise and improve personal balance in environment [26]. PD patients with freezing of gait display reduced network connections in $\mathrm{VN}[27,28]$. Thus, the improved VN function may explain the better performance in BBS in PD after Tai Chi training. We also observed that changes of DMN connectivity were related 
to the improvement in UPDRS total score and UPDRSIII score. The DMN includes the hippocampus, parahippocampal, fusiform and angular gyrus, the precuneus and the middle temporal gyrus [25]. Precuneus is one of the functional hub regions of DMN, and its interactions with sensorimotor network are positively associated with motor performances [29]. Thus, the improvement of DMN connectivity may explain the improved motor function of PD patients after Tai Chi training, since the connection of precuneus to motor areas might be associated with processes of motor mental imagery and planning [29].

In this study, proinflammatory cytokines were downregulated after Tai Chi training. Among them, the decreased IL-1 $\beta$ was correlated with improved BBS score. Inflammation plays an important role in the pathogenesis and disease progression of PD [15]. A meta-analysis study of inflammatory cytokines in PD has demonstrated significantly higher blood levels of IL-1 $\beta$ compared with healthy controls [30]. IL-1 $\beta$ plays an important role in different neurobiological processes, such as neuroinflammation, neurotoxicity, and host defense. Therefore, this cytokine has been linked to both acute and chronic neurodegenerative conditions [31]. Lipopolysaccharide (LPS) induces PD symptoms by stimulating IL- $1 \beta$ in wild-type animals, suggesting that IL-1 $\beta$ may contribute to the initiation or progression of PD [31]. Decreased IL-1 $\beta$, which indicates reduced inflammation, may explain the improved BBS performance of PD patients after Tai Chi training. The tendency of decreased IL- $1 \beta$ by Tai Chi training but increased IL- $1 \beta$ by brisk walking training (6-month, $P=0.053$; 12-month, $P=0.096$; Additional file 2: Table S3) may explain the superiority of Tai Chi over brisk walking in improving the balance of PD patients.

In addition, the dysregulation of metabolites and metabolic pathways in PD revealed here was mainly associated with amino acid metabolism (pipecolic acid, $L$-fucose, and arginine biosynthesis), energy metabolism ( $L$-malic acid, 3-phosphoglyceric acid, urea cycle, TCA cycle and beta oxidation of very-long-chain fatty acids) and neurotransmitter metabolism (adenosine) [32]. $L$-arginine participates in the synthesis of nitric oxide and can affect oxidative stress and energy metabolism, playing a key role in the pathogenesis of PD [33]. Deficiency of TCA cycle enzymes and dysfunction of mitochondria, which regulate neuroinflammation and neurodegeneration, have also been observed in PD [34]. The coupling of adenosine with its specific receptors acts as an upstream neuromodulator for neurotransmitters such as acetylcholine, glutamate, $\gamma$-amino-butyric acid, and dopamine that is implicated in the modulation of multiple body functions [35]. Our results indicated improved amino acid metabolism, energy metabolism and neurotransmitter metabolism in PD patients after Tai Chi training.

HIP2 is an E2 ubiquitin-conjugating enzyme related to protein cleavage via the ubiquitin-proteasome system (UPS) pathway [36]. Impaired UPS system is related to protein aggregation, causing inflammation and abnormal oxidation [37]. Reduction of HIP2 expression leads to impairment of spontaneous motor function and increased vulnerability to dopaminergic degeneration in PD models [21]. In our previous study, HIP2 mRNA expression was downregulated in $20 \mathrm{PD}$ patients and then elevated after one-year Tai Chi training with improved motor function [21]. Here, the results further confirmed that Tai Chi training could reverse the downregulation of HIP2 mRNA in a larger PD cohort, and this change was correlated with the improvement of motor function in PD patients after Tai Chi training, suggesting that Tai Chi training can decrease the vulnerability to dopaminergic degeneration in PD.

These lines of fMRI and blood biomarker evidence suggest enhanced brain network function, reduced inflammation, improved amino acid metabolism, energy metabolism and neurotransmitter metabolism, as well as decreased vulnerability to dopaminergic degeneration in PD after Tai Chi training.

To our interest, one-year Tai Chi training decreased the UPDRS-III score compared to baseline (baseline $25.20 \pm 17.50 v s$ one-year $19.10 \pm 9.56$ ), while the motor symptoms became worse in the brisk walking group (baseline $17.50 \pm 7.01$ vs one-year $23.10 \pm 7.81$ ) and the control group (baseline $19.30 \pm 4.87$ vs one-year $30.70 \pm 7.35)$. Since there was no statistical difference in the change of LEDD among the three groups $(P=0.939)$, which could exclude the impact of LEDD, this result indicated that Tai Chi training may have disease-modifying effects on PD.

\section{Limitations}

There were some limitations in our study. First, the number of participants in our study was not large enough. Validity might be lost because of the small sample size. Therefore, larger-size cohort studies are warranted. Second, the dropout rate in the brisk walking and control groups could not be ignored. Since our study observed long-term effects of Tai Chi for one year, the follow-up duration is relatively too long to keep a low drop-out rate. Besides, patients in the Tai Chi class were willing to stick to Tai Chi training since they had benefited from it. Third, we recruited mainly early-stage PD patients. Tai Chi training has a high demand for strength and balance of the lower limbs. If patients have remarkably impaired balance, they would have an increased risk of falling. Thus, early-stage PD patients were recruited to protect 
them from falling and injuries during training. Besides, early-stage PD patients have less difficulty in mobility and are more likely to meet the requirements for training, which could ensure the quality of training. To further confirm the positive effects of Tai Chi on balance, future studies of Tai Chi training in moderate PD patients are warranted.

\section{Conclusions}

Our study revealed that long-term Tai Chi training improves motor function, especially gait and balance, in PD patients. Enhanced brain network function, reduced inflammation, improved amino acid metabolism, energy metabolism and neurotransmitter metabolism, as well as decreased vulnerability to dopaminergic degeneration may be mechanisms underlying the effects of Tai Chi training.

\begin{abstract}
Abbreviations
BBS: Berg Balance scale; DMN: Default mode network; FDR: False discovery rate; fMRI: Functional magnetic resonance imaging; GM-CSF: Granulocytemacrophage colony stimulating factor; HIP2: Huntingtin interaction protein 2; L: Interleukin; LEDD: Levodopa equivalent daily dosage; MIP: Macrophage inflammatory protein; PD: Parkinson's disease; TUG: Timed up and go; UPDRS: Unified Parkinson's Disease Rating Scale; UPS: Ubiquitin proteasome system; VN: Visual network
\end{abstract}

\section{Supplementary Information}

The online version contains supplementary material available at https://doi. org/10.1186/s40035-022-00280-7.

\section{Additional file 1: Protocol. \\ Additional file 2: Table S1-S10. Table S1 Clinical assessments of motor symptoms among Tai Chi, Brisk Walking and Control group; Table S2 The association between clinical improvements and the switch rate of brain networks; Table S3 Intergroup comparison of cytokines among Tai Chi group, Brisk Walking group and Control group; Table S4 The association between changes of cytokines and rating scales; Table S5 Intergroup comparison of metabolites among Tai Chi group, Brisk Walking group and Control group; Table S6 Multivariate analysis of metabolomics and rating scale; Table S7 Pathway analysis of metabolites; Table S8 Enrich- ment analysis of metabolites; Table S9 Associations between Pathway/ Enrichment Analysis of Metabolomics and clinical presentations among 3 Groups; Table S10 Associations between HIP2 mRNA level and clinical presentations in Tai Chi group.}

Additional file 3: Fig. S1. Pathway analysis in Tai Chi group relative to the Control group from baseline to six-month visit; Fig. S2 Pathway analysis in Tai Chi group relative to the Control group from six-month visit to oneyear visit; Fig. S3 Pathway analysis in Tai Chi group relative to the Control group from baseline to one-year visit; Fig. S4 Enrichment analysis in Tai Chi group relative to the Control group from baseline to six-month visit; Fig. S5 Enrichment analysis in Tai Chi group relative to the Control group from six-month visit to one-year visit; Fig. S6 Enrichment analysis in Tai Chi group relative to the Control group from baseline to one-year visit; Fig. S7 Comparison of the change of HIP2 mRNA level in the 3 groups.

\section{Acknowledgements}

We thank all coaches of Sino Taiji of Fuxing International for their Tai Chi training to PD patients. We also thank all patients who participated in this study.

\section{Authors' contributions}

GL and PH performed clinical assessment, ran statistical analysis and drafted the manuscript. SSC, YCH and XS performed clinical assessment. QYJ performed fMRI scan. PH performed gait assessment. GYH and BYL made FMRI analysis. YXL made FMRI data management. JX and ZW participated in study design. SDC and YYT performed patient recruitment and diagnosis. SDC designed the study, supervised the study, double-checked the statistical analysis and revised the manuscript. All authors read and approved the final manuscript.

\section{Funding}

This work was supported by grants from the National Natural Science Foundation of China $(81430022,91332107,81371407,81801267$, 31771174, 82151303), Shanghai Municipal Science and Technology Major Project (2018SHZDZX05) and Shanghai Municipal Education Commission (2017NKX001).

\section{Availability of data and materials}

The datasets used and analyzed during the current study are available from the corresponding author on reasonable request.

\section{Declarations}

\section{Ethical approval and consent to participate}

This study was approved by Ruijin Hospital Ethic Committee of Shanghai Jiao Tong University School of Medicine, and all participants had given written informed consent.

\section{Consent for publication}

Not applicable.

\section{Competing interests}

The authors declare that they have no competing interests.

\section{Author details}

${ }^{1}$ Department of Neurology and Institute of Neurology, Ruijin Hospital Affiliated to Shanghai Jiao Tong University School of Medicine, Shanghai 200025, China. ${ }^{2}$ Institute of Neuroscience, State Key Laboratory of Neuroscience, Key Laboratory of Primate Neurobiology, CAS Center for Excellence in Brain Science and Intelligence Technology, Shanghai Institute for Biological Sciences, Chinese Academy of Sciences, Shanghai 200031, China. ${ }^{3}$ Shanghai Key Laboratory for Bone and Joint Diseases, Shanghai Institute of Orthopedics and Traumatology, Ruijin Hospital Affiliated to Shanghai Jiao Tong University School of Medicine, Shanghai 200003, China. ${ }^{4}$ School of Psychological and Cognitive Sciences, Beijing Key Laboratory of Behavior and Mental Health, IDG/McGovern Institute for Brain Research, Peking-Tsinghua Center for Life Sciences, Peking University, Beijing 100871, China.

Received: 14 September 2021 Accepted: 5 January 2022 Published online: 07 February 2022

\section{References}

1. Kalia LV, Lang AE. Parkinson's disease. Lancet. 2015;386:896-912.

2. Ashburn A, Stack E, Pickering RM, Ward CD. A community-dwelling sample of people with Parkinson's disease: characteristics of fallers and non-fallers. Age Ageing. 2001;30:47-52.

3. Armstrong MJ, Okun MS. Diagnosis and treatment of Parkinson disease: a review. JAMA. 2020;323:548-60.

4. Earhart GM, Falvo MJ. Parkinson disease and exercise. Compr Physiol. 2013;3:833-48.

5. Van Der Kolk NM, King LA. Effects of exercise on mobility in people with Parkinson's disease. Mov Disord. 2013;28:1587-96.

6. Li F, Harmer P, Fitzgerald K, Eckstrom E, Stock R, Galver J, et al. Tai chi and postural stability in patients with Parkinson's disease. N Engl J Med. 2012;366:511-9.

7. Mak MKY, Wong-Yu ISK. Six-month community-based brisk walking and balance exercise alleviates motor symptoms and promotes functions in 
people with Parkinson's disease: a randomized controlled trial. J Parkinsons Dis. 2021;11:1431-41.

8. Rios Romenets S, Anang J, Fereshtehnejad SM, Pelletier A, Postuma R. Tango for treatment of motor and non-motor manifestations in Parkinson's disease: a randomized control study. Complement Ther Med. 2015;23:175-84.

9. Lan C, Chen SY, Lai JS, Wong AM. Tai chi chuan in medicine and health promotion. Evid Based Complement Altern Med. 2013;2013:502131.

10. Field T. Tai Chi research review. Complement Ther Clin Pract. 2011;17:141-6.

11. Li F, Harmer P. Economic evaluation of a Tai Ji Quan intervention to reduce falls in people with Parkinson disease, Oregon, 2008-2011. Prev Chronic Dis. 2015;12:E120.

12. Mahalakshmi B, Maurya N, Lee SD, Bharath Kumar V. Possible neuroprotective mechanisms of physical exercise in neurodegeneration. Int J Mol Sci. 2020;21.

13. Zhou M, Liao H, Sreepada LP, Ladner JR, Balschi JA, Lin AP. Tai Chi improves brain metabolism and muscle energetics in older adults. $J$ Neuroimaging. 2018;28:359-64.

14. Liu J, Tao J, Liu W, Huang J, Xue X, Li M, et al. Different modulation effects of Tai Chi Chuan and Baduanjin on resting-state functional connectivity of the default mode network in older adults. Soc Cogn Affect Neurosci. 2019;14:217-24

15. Jiang $T, L i G, X u J, G a o ~ S$, Chen X. The challenge of the pathogenesis of Parkinson's disease: Is autoimmunity the culprit? Front Immunol. 2018:9:2047.

16. Hatano T, Saiki S, Okuzumi A, Mohney RP, Hattori N. Identification of novel biomarkers for Parkinson's disease by metabolomic technologies. J Neurol Neurosurg Psychiatry. 2016;87:295-301.

17. Scherzer CR, Eklund AC, Morse LJ, Liao Z, Locascio JJ, Fefer D, et al. Molecular markers of early Parkinson's disease based on gene expression in blood. Proc Natl Acad Sci U S A. 2007;104:955-60.

18. Karlsson MK, Sharma P, Aasly J, Toft M, Skogar O, Sæbø S, et al. Found in transcription: accurate Parkinson's disease classification in peripheral blood. J Parkinsons Dis. 2013:3:19-29.

19. Calligaris R, Banica M, Roncaglia P, Robotti E, Finaurini S, Vlachouli C, et al. Blood transcriptomics of drug-naïve sporadic Parkinson's disease patients. BMC Genom. 2015:16:876.

20. Grünblatt E, Mandel S, Jacob-Hirsch J, Zeligson S, Amariglo N, Rechavi $G$, et al. Gene expression profiling of parkinsonian substantia nigra pars compacta; alterations in ubiquitin-proteasome, heat shock protein, iron and oxidative stress regulated proteins, cell adhesion/cellular matrix and vesicle trafficking genes. J Neural Transm (Vienna). 2004;111:1543-73.

21. Su J, Huang P, Qin M, Lu Q, Sang X, Cai Y, et al. Reduction of HIP2 expression causes motor function impairment and increased vulnerability to dopaminergic degeneration in Parkinson's disease models. Cell Death Dis. 2018:9:1020.

22. Hughes AJ, Daniel SE, Kilford L, Lees AJ. Accuracy of clinical diagnosis of idiopathic Parkinson's disease: a clinico-pathological study of 100 cases. J Neurol Neurosurg Psychiatry. 1992;55:181-4.

23. Postuma RB, Berg D, Stern M, Poewe W, Olanow CW, Oertel W, et al. MDS clinical diagnostic criteria for Parkinson's disease. Mov Disord. 2015:30:1591-601.

24. Pedersen M, Zalesky A, Omidvarnia A, Jackson GD. Multilayer network switching rate predicts brain performance. Proc Natl Acad Sci U S A. 2018;115:13376-81.

25. Pedersini CA, Guàrdia-Olmos J, Montalà-Flaquer M, Cardobi N, SanchezLopez J, Parisi G, et al. Functional interactions in patients with hemianopia: A graph theory-based connectivity study of resting fMRI signal. PLoS One. 2020;15:e0226816

26. Assländer $L$, Hettich $G$, Mergner T. Visual contribution to human standing balance during support surface tilts. Hum Mov Sci. 2015:41:147-64.

27. Ruan X, Li Y, Li E, Xie F, Zhang G, Luo Z, et al. Impaired topographical organization of functional brain networks in Parkinson's disease patients with freezing of gait. Front Aging Neurosci. 2020;12:580564.

28. Rogge AK, Röder B, Zech A, Hötting K. Exercise-induced neuroplasticity: Balance training increases cortical thickness in visual and vestibular cortical regions. Neuroimage. 2018;179:471-9.

29. Thibes RB, Novaes NP, Lucato LT, Campanholo KR, Melo LM, Leite CC, et al. Altered functional connectivity between precuneus and motor systems in Parkinson's disease patients. Brain Connect. 2017;7:643-7.
30. Qin XY, Zhang SP, Cao C, Loh YP, Cheng Y. Aberrations in peripheral inflammatory cytokine levels in Parkinson disease: a systematic review and meta-analysis. JAMA Neurol. 2016;73:1316-24.

31. Saghazadeh A, Ferrari CC, Rezaei N. Deciphering variability in the role of interleukin-1 $\beta$ in Parkinson's disease. Rev Neurosci. 2016;27:635-50.

32. Shao Y, LeW. Recent advances and perspectives of metabolomics-based investigations in Parkinson's disease. Mol Neurodegener. 2019;14:3.

33. Virarkar M, Alappat L, Bradford PG, Awad AB. L-arginine and nitric oxide in CNS function and neurodegenerative diseases. Crit Rev Food Sci Nutr. 2013:53:1157-67.

34. Bose A, Beal MF. Mitochondrial dysfunction in Parkinson's disease. J Neurochem. 2016;139(Suppl 1):216-31.

35. Soliman AM, Fathalla AM, Moustafa AA. Adenosine role in brain functions: pathophysiological influence on Parkinson's disease and other brain disorders. Pharmacol Rep. 2018;70:661-7.

36. Chen ZJ, Niles EG, Pickart CM. Isolation of a cDNA encoding a mammalian multiubiquitinating enzyme (E225K) and overexpression of the functional enzyme in Escherichia coli. J Biol Chem. 1991;266:15698-704.

37. Bence NF, Sampat RM, Kopito RR. Impairment of the ubiquitin-proteasome system by protein aggregation. Science. 2001;292:1552-5.
Ready to submit your research? Choose BMC and benefit from:

- fast, convenient online submission

- thorough peer review by experienced researchers in your field

- rapid publication on acceptance

- support for research data, including large and complex data types

- gold Open Access which fosters wider collaboration and increased citations

- maximum visibility for your research: over $100 \mathrm{M}$ website views per year

At BMC, research is always in progress.

Learn more biomedcentral.com/submissions 